\title{
Diversidad genética de Mangifera indica (Anacardiaceae) en Valencia, Córdoba, Colombia, usando marcadores microsatélites
}

\section{Genetic diversity of Mangifera indica (Anacardiaceae) in Valencia, Córdoba, Colombia, using microsatellite markers}

Universidad de Córdoba, Facultad de Ciencias Básicas, Programa de Biología, Montería-Córdoba, ColombiaCarrera 6 No. 76-103, 230002 Montería, Colombia.

2 Autor para la correspondencia: epardop@correo.unicordoba.edu.co

Recibido: 23 de septiembre de 2017 Revisado: 17 de octubre de 2017. Aceptado: 16 de enero de 2018.

Primero en línea: 29 de mayo de 2018. Publicado: 1 de julio de 2018.

\section{Citar como:}

Guerra, M., R. Ruiz y E. Pardo. 2018. Diversidad genética de Mangifera indica (Anacardiaceae) en Valencia, Córdoba, Colombia, usando marcadores microsatélites. Acta Botanica Mexicana 124: 105-116. DOI: 10.21829/ abml24.2018.1285

DOI:

10.21829/abm124.2018.1285
Martha Guerra' (D), Rosalba Ruiz' (D), Enrique Pardo',2 (D)

\section{Resumen:}

Antecedentes y Objetivos: El mango (Mangifera indica) pertenece a la familia Anacardiaceae y es nativa del sur de Asia. Actualmente ha alcanzado una gran distribución por su desarrollo en climas cálidos, y su adaptación a una amplia gama de condiciones ambientales. El conocimiento de la diversidad genético poblacional del germoplasma de mango en Valencia, Córdoba, Colombia, permitiría seleccionar variedades y poblaciones promisorias para emplearse en los programas de mejoramiento genético del país. El objetivo del presente trabajo fue determinar la diversidad genética de una población de Mangifera indica en Valencia empleando 12 marcadores microsatélites.

Métodos: El estudio se realizó con hojas de Mangifera indica colectadas en el municipio de Valencia deshidratadas con silica gel. El análisis de los individuos se realizó utilizando 12 marcadores moleculares microsatélites. Empleando diferente software (GENALEX, CERVUS, FSTAT y MEGA 7) se determinó: número de alelos, número efectivo de alelos, heterocigosidad observada y esperada, distancia genética y equilibrio de Hardy-Weinberg, contenido de información polimórfica, índices de fijación $\mathrm{F}_{\mathrm{IS}}$, $\mathrm{F}_{\mathrm{IT}}$ y $\mathrm{F}_{\mathrm{ST}}$, $\mathrm{y}$ un dendrograma.

Resultados clave: Todos los microsatélites analizados fueron polimórficos. Se detectaron entre 5 y 12 alelos, con un promedio de 7 alelos por locus y un total de 84 . El número efectivo de alelos promedio fue 4.551. Los valores del PIC oscilaron entre 0.86 y 0.49 para los marcadores MiIIHR23 y MiIIHR34 respectivamente. La prueba de Hardy-Weinberg indicó que la población estaba en desequilibrio $(\mathrm{p}<0.05)$ para los 12 marcadores. El índice de fijación reveló un exceso de homocigotos. El promedio de heterocigosidad, observada y esperada, fue de 0.355 y 0.748 respectivamente.

Conclusiones: La población analizada presentó alta diversidad genética y los marcadores resultaron muy informativos, atendiendo al PIC.

Palabras clave: alelos, contenido de información polimórfica, distancia genética, equilibrio de HardyWeinberg.

\section{ABSTRACT:}

Background and Aims: Mango (Mangifera indica) belongs to the Anacardiaceae family and is native to South Asia. It has currently reached a large distribution because of its development in warm climates, and its adaptation to a wide range of environmental conditions. Knowledge of the population genetic diversity of mango germplasm in Valencia, Córdoba, Colombia, would allow for selection of promising mango varieties and populations for use in the country's breeding programs. The aim of the present work was to determine the genetic diversity of a population of Mangifera indica in Valencia using 12 microsatellite markers.

Methods: The study was conducted with leaves of Mangifera indica collected in the city of Valencia and dehydrated with silica gel. The analysis of individuals was performed using 12 microsatellite molecular markers. Employing different software (GENALEX, CERVUS, FSTAT and MEGA 7), were determined: number of alleles, effective number of alleles, heterozygosity observed and expected, genetic distance, Hardy-Weinberg equilibrium, polymorphic information content, fixation indices $\mathrm{F}_{\mathrm{IS}}, \mathrm{F}_{\mathrm{IT}}, \mathrm{F}_{\mathrm{ST}}$ and a dendrogram. Key results: All microsatellites used were polymorphic. Between 5 to 12 alleles were detected, with an average of 7 alleles per locus and a total of 84 . The average effective number of alleles was 4.551. PIC values ranged between 0.86 and 0.49 for MiIIHR23 and MiIIHR34 respectively. The Hardy-Weinberg test indicated that the population was in disequilibrium $(\mathrm{p}<0.05)$ for the 12 markers. The fixation index revealed an excess of homozygotes. The average of the observed and expected heterozygosity was 0.355 and 0.748 respectively.

Conclusions: The analyzed population presented high genetic diversity and the markers were very informative, attending the PIC.

Key words: alleles, distance genetics, Hardy-Weinberg equilibrium, polymorphic information content. 


\section{INTRODUCCIÓN}

El mango (Mangifera indica L.) es una planta perteneciente a la familia Anacardiaceae, nativa del sur de Asia, especialmente del Oriente de India; actualmente ha alcanzado una gran distribución por su cultivo en alrededor de 100 países (Galán, 2009), debido a su desarrollo en climas cálidos, y su adaptación a una gama de condiciones ambientales amplias (Asohofrucol y Corpoica, 2013).

Mangifera indica es una de las especies más apreciadas a nivel mundial, destacándose por la propagación y aprovechamiento del fruto, su utilidad para fines comerciales (Human y Rheeder, 2004) y farmacéuticos (Márquez et al., 2010), y por sus propiedades organolépticas y nutricionales (Kessel, 2008). Por sus atributos, $M$. indica es considerada de gran importancia comercial y con potencial económico (Galán, 2009), siendo cultivada en casi todas las zonas tropicales y subtropicales, principalmente en India, Brasil y México. La vasta distribución geográfica y ambiental de esta especie, ha sido un factor que ha propiciado la amplia diversidad genética de la misma, generando genotipos que exhiben diferencias notorias, particularmente en caracteres del fruto (García et al., 2010).

En Colombia, el cultivo de mango se produce en alrededor de 23 mil hectáreas (Espinal-García et al., 2005), presentes en 16 departamentos, y cuenta con aproximadamente 100 variedades (Espinal-García et al., 2005). Los mangos criollos son variedades de tamaño pequeño, reproducción poliembriónica, mayor vigor en las plantas e igual calidad que la planta madre (Vieccelli et al., 2016). Se considera que la mayoría de los cultivares de mango criollo tienen un origen poliembriónico (Vieccelli et al., 2016).

Los cultivares de mango, al igual que otras especies de árboles frutales, son identificados con base en rasgos morfológicos sustentados en descriptores (IPGRI, 2006). Sin embargo, la determinación basada exclusivamente en caracteres morfológicos es inexacta debido a la influencia de los factores ambientales y al número limitado de caracteres distinguibles. De esta manera, la identificación molecular de los cultivares de mango se ha llevado a cabo con diferentes sistemas moleculares como isoenzimas
(Degani et al., 1990; Aron et al., 1997), repeticiones en tándem de número variable (VNTR) (Adato et al., 1995; Krishna y Singh, 2007; Begum et al., 2012; Sennhenn et al., 2014; Wang et al., 2016), polimorfismos de longitud en fragmentos amplificados (AFLPs) (Eiadthong et al., 2000; Kashkush et al., 2001; Yamanaka et al., 2006; Santos et al., 2008; Gálvez-López et al., 2009), ADN polimórficos amplificados al azar (RAPDs) (Souza et al., 2011; Samal et al., 2012; Hossain et al., 2016; Pruthvish y Chikkaswamy, 2016; Galal et al., 2017) y repetición de secuencias discretas (SSRs) (Surapaneni et al., 2013; Ravishankar et al., 2015; Azmat et al., 2016; Bajpai et al., 2016; dos Santos Alves et al., 2016; Nazish et al., 2017). Los resultados revelan diferencias entre las accesiones de mango sin importar el sistema de marcador utilizado, su origen geográfico o su situación genética (cultivares, razas autóctonas o variedades).

Las repeticiones de secuencia simple (SSRs) constituyen una de las herramientas más específicas, eficaces y rápidas para analizar la diversidad genética de las especies (Azofeifa-Delgado, 2006), se trata de secuencias de ADN de 1 a 6 nucleótidos dispuestos en grupo, distribuidos en todo el genoma y cuya herencia es segregante (Becerra y Paredes, 2000). Estos marcadores presentan múltiples ventajas como codominancia, multialelismo y alta heterocigosidad; además, requieren una cantidad mínima de ADN para su estudio y realizan una discriminación precisa entre individuos altamente emparentados gracias a su alto nivel polimórfico (González, 2003).

El conocimiento sobre la diversidad genética de las poblaciones de mango en Colombia es pobre, destacándose el estudio realizado en seis poblaciones de Colombia (Díaz-Matallana et al., 2009), por lo que se desconoce la diversidad genética poblacional de Mangifera indica a nivel de Valencia, Córdoba. En este sentido, esta situación afecta al sector agrícola y a la comunidad científica ante la carencia de información para seleccionar variedades y poblaciones de mango promisorias para el mejoramiento genético o selección de cultivares con mayor productividad.

Por lo anterior, este trabajo tiene como objetivo determinar en el municipio de Valencia, departamento de 
Córdoba, la diversidad genética de 35 accesiones de Mangifera indica, empleando 12 marcadores microsatélites, como requerimiento básico para los programas de mejoramiento de mango, conservación y selección de materiales promisorios (Krishna y Singh, 2007), para la industria alimentaria a nivel nacional.

\section{Materiales y Métodos}

\section{Material vegetal}

El material vegetal estudiado fue colectado en cinco fincas del municipio de Valencia, en el departamento de Córdoba, Colombia (Fig. 1). Se muestrearon 35 accesiones distribuidas en subpoblaciones de la siguiente forma: Villanueva: $8^{\circ} 20^{\prime} 27.97^{\prime \prime} \mathrm{N} ; 7^{\circ} 07^{\prime} 0848^{\prime \prime} \mathrm{O}(\mathrm{n}=6)$,
Valencia: $8^{\circ} 15^{\prime} 25.77 " \mathrm{~N} ; 7^{\circ} 10^{\prime} 27.79^{\prime \prime} \mathrm{O}(\mathrm{n}=8)$, El Reposo: $8^{\circ} 10^{\prime} 41.44 " \mathrm{~N} ; 76^{\circ} 10^{\prime \prime} 13.51 " \mathrm{O}(\mathrm{n}=6)$, San Rafael del Pirú: $8^{\circ} 11^{\prime} 06.89^{\prime \prime N} ; 76^{\circ} 16^{\prime} 40.56^{\prime \prime O}(\mathrm{n}=8)$, Santo Domingo: $8^{\circ} 08^{\prime} 36.88^{\prime \prime} \mathrm{N} ; 7^{\circ} 19^{\prime} 34.29^{\prime \prime} \mathrm{O}(\mathrm{n}=7)$.

Las accesiones muestreadas fueron deshidratadas con silica gel y mantenidas bajo las condiciones del Herbario HUC de la Universidad de Córdoba.

\section{Extracción de ADN genómico}

Para cada muestra se colocaron fragmentos de tejido foliar seco en una cápsula de porcelana con nitrógeno líquido, se maceraron hasta obtener un polvo fino y se guardaron a $-20{ }^{\circ} \mathrm{C}$ hasta su empleo.

La extracción del ADN genómico se llevó a cabo en $40 \mathrm{mg}$ del material macerado, usando el Dneasy Plant

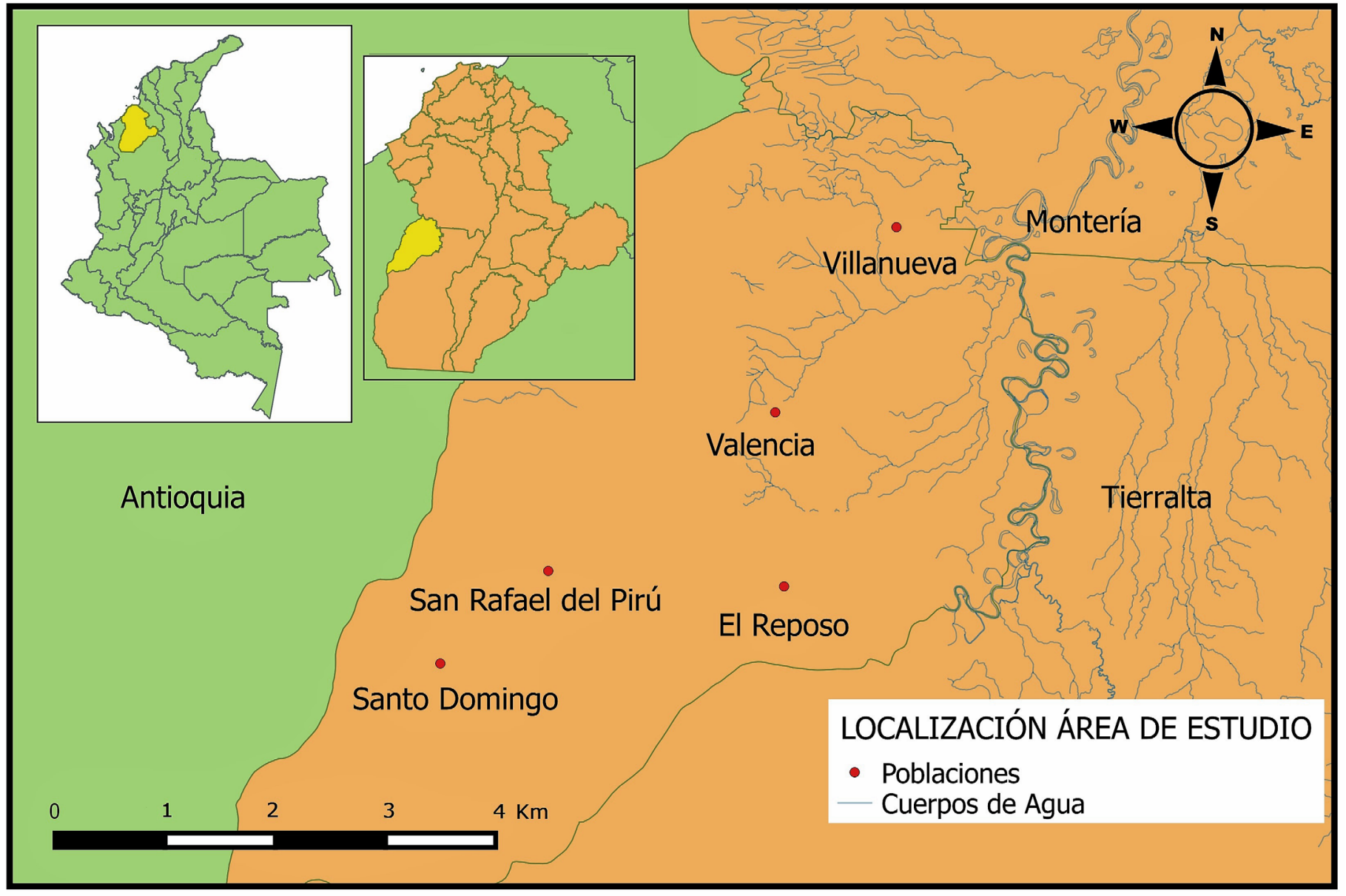

Figura 1: Mapa de ubicación del municipio Valencia, departamento de Córdoba, Colombia. 
Mini Kit de extracción de QIAGEN ${ }^{\circledR}$ (QIAGEN Inc., Valencia, CA, USA).

\section{Análisis genético de repeticiones de secuen- cia simple (SSRs)}

La concentración de ADN se determinó mediante un espectrofotómetro NanoDrop ${ }^{\circledR}$ ND-1000 (Thermo Fisher Scientific, Wilmington, EUA) a 260 y $280 \mathrm{~nm}$ de longitud de onda, como lo señalan Valadez y Kahl (2000).

Se emplearon 12 loci microsatélites utilizados por Ravishankar et al., (2011). La amplificación de los mismos se realizó mediante PCR en un volumen de mezcla de reacción de $12 \mu 1$ que incluyó $0.24 \mu \mathrm{l}$ de dNTPs $10 \mu \mathrm{M}$, $1.8 \mu \mathrm{l}$ de amortiguador $10 \mathrm{X}, 0.72 \mu \mathrm{lde} \mathrm{MgCl}_{2} 1.5 \mu \mathrm{M}$, $0.12 \mu \mathrm{l}$ de cebadores específicos (forward y reverse) 0.5 $\mu \mathrm{M}, 0.12 \mu \mathrm{l}$ de enzima Taq polimerasa a una concentración de $0.05 \mathrm{U} / \mu \mathrm{l}, 2 \mu \mathrm{l}$ de ADN genómico a una concentración de $3.33 \mathrm{ng} / \mu \mathrm{l}$ y $6.88 \mu \mathrm{l}$ de agua estéril.

La reacción de PCR se realizó en un Termociclador Bio-Rad T100 ${ }^{\mathrm{TM}}$ (Applied Biosystems, Foster City, USA) y consistió de una fase de desnaturalización de 95 ${ }^{\circ} \mathrm{C}$ durante $3 \mathrm{~min}$, una segunda fase de 35 ciclos de 30 $\mathrm{s}$ de desnaturalización a $95{ }^{\circ} \mathrm{C}, 30 \mathrm{~s}$ a la temperatura de alineamiento (Cuadro 1), seguido por una tercera fase de extensión a $72{ }^{\circ} \mathrm{C}$ por 1 min y finalmente, una fase de extensión de 5 min a $72{ }^{\circ} \mathrm{C}$.

Los productos de PCR se separaron mediante electroforesis vertical en gel de poliacrilamida a $10 \%$ (acrilamida: bisacrilamida, 29:1) desnaturalizado (6 mol L-1 de urea), en una cámara Mini-Protean II Biorad ${ }^{\circledR}$

Cuadro 1: Microsatélites tipificados de Mangifera indica L. (Ravishankar et al., 2011), secuencia de iniciadores, numero de alelos y rango alélico (pb).

\begin{tabular}{|c|c|c|c|c|}
\hline Marcador & Secuencia de iniciadores & $\begin{array}{c}\text { Temperatura de } \\
\text { alineamiento }\left({ }^{\circ} \mathbf{C}\right)\end{array}$ & $\begin{array}{l}\text { No. de } \\
\text { alelos }\end{array}$ & Rango alélico (pb) \\
\hline \multirow[t]{2}{*}{ MiIIHR12 } & F: GCCCCATCAATACGATTGTC & 55 & 9 & $154-185$ \\
\hline & R: ATTTCCCACCATTGTCGTTG & & & \\
\hline \multirow[t]{2}{*}{ MiIIHR13 } & F: CCCAGTTCCAACATCATCAG & 55 & 5 & $189-201$ \\
\hline & R: TTCCTCTGGAAGAGGGAAGA & & & \\
\hline \multirow[t]{2}{*}{ MiIIHR15 } & F: TAACCATTCGGCATCCTCT & 55 & 5 & 153-164 \\
\hline & R: TGTGATAGAATGGCAAAAGAA & & & \\
\hline \multirow[t]{2}{*}{ MiIIHR17 } & F: GCTTGCTTCCAACTGAGACC & 57 & 7 & $258-294$ \\
\hline & R: GCAAAATGCTCGGAGAAGAC & & & \\
\hline \multirow[t]{2}{*}{ MiIIHR18 } & F: TCTGACGTCACCTCCTTTCA & 55 & 5 & $170-181$ \\
\hline & R: ATACTCGTGCCTCGTCCTGT & & & \\
\hline \multirow[t]{2}{*}{ MiIIHR19 } & F: TGATATTTTCAGGGCCCAAG & 56 & 7 & $192-224$ \\
\hline & R: AAATGGCACAAGTGGGAAAG & & & \\
\hline \multirow[t]{2}{*}{ MiIIHR23 } & F: TCTGACCCAACAAAGAACCA & 55 & 12 & $112-150$ \\
\hline & R: TCCTCCTCGTCCTCATCATC & & & \\
\hline \multirow[t]{2}{*}{ MiIIHR24 } & F: GCTCAACGAACCCAACTGAT & 55 & 8 & $245-274$ \\
\hline & R: CCAGCATTCAATGAAGAAGTT & & & \\
\hline \multirow[t]{2}{*}{ MiIIHR26 } & F: GCGAAAGAGGAGAGTGCAAG & 56 & 8 & $135-156$ \\
\hline & R: TCTATAAGTGCCCCCTCACG & & & \\
\hline \multirow[t]{2}{*}{ MiIIHR31 } & F: TTCTGTTAGTGGCGGTGTTG & 56 & 5 & $212-236$ \\
\hline & R: CACCTCCTCCTCСТCСТCTT & & & \\
\hline \multirow[t]{2}{*}{ MiIIHR34 } & F: CTGAGTTTGGCAAGGGAGAG & 55 & 5 & $227-243$ \\
\hline & R: TTGATCCTTCACCACCATCA & & & \\
\hline \multirow[t]{2}{*}{ MiIIHR36 } & F: TCTATAAGTGCCCCCTCACG & 56 & 8 & $222-250$ \\
\hline & R: ACTGCCACCGTGGAAAGTAG & & & \\
\hline Promedio total & & - & 7 & - \\
\hline
\end{tabular}


(Applied Biosystems, Foster City, EUA) (Tsang et al., 1986). Las bandas se visualizaron por tinción con nitrato de plata (Qiu et al., 2012), utilizando DNA ladder, donde el rango del marcador de peso molecular utilizado osciló entre 50 y $500 \mathrm{pb}$. Los geles fueron fotografiados con una cámara CANON (ELPH180 IS, Tokio, Japón) y la determinación del tamaño alélico se efectuó mediante el programa ImageJ (Abràmoff et al., 2004) donde por pixelaje de las bandas amplificadas, se determinaron los tamaños alélicos utilizando el software Past 3.14 (Hammer et al., 2001).

\section{Análisis estadístico}

Mediante el software GenAlEx versión 6.5 (Peakall y Smouse, 2006) se calculó para cada locus: número de alelos observados, número efectivo de alelos, valores de heterocigosidad observada y esperada (Ho y He), distancia genética y equilibrio de Hardy-Weinberg. El PIC (Contenido de Información Polimórfica) de cada locus microsatélite se estimó mediante el programa CERVUS versión 3.0.7 (Kalinowski et al., 2007). Los índices de fijación de Wright: $\mathrm{F}_{\mathrm{IS}}, \mathrm{F}_{\mathrm{IT}}$ y $\mathrm{F}_{\mathrm{ST}}$, se calcularon mediante el programa FSTAT versión 2.9.3.2 (Goudet et al., 2002). El dendrograma se construyó mediante el método UPGMA con un bootstrap de $80 \%$, empleando el programa MEGA versión 7 (Kumar et al., 2016). Asimismo, para la identificación de duplicados se utilizó el software Excel Microsatellite Toolkit (Park, 2001).

\section{Resultados}

En total, 84 alelos fueron observados para la población de mangos en Valencia, Córdoba, Colombia, con un número promedio de siete alelos en el germoplasma del mango. El locus MiIIHR23 presentó el mayor número (12) de alelos y los loci que presentaron el menor número (5) de alelos fueron MiIIHR13, MiIIHR15, MiIIHR18, MiIIHR31 y MiIIHR34 (Cuadro 1).

El promedio del número efectivo de alelos fue de 4.5, el locus con el mayor número efectivo (7.9) de alelos fue MiIIHR23 y MiIIHR34 presentó el menor número efectivo (2.1) de alelos (Cuadro 2).
Cuadro 2: Parámetros básicos de diversidad genética calculados para el germoplasma de Mangifera indica L. en Valencia, Córdoba, Colombia. Número efectivo de alelos: $\mathrm{Na}$, heterocigosidad observada: Ho, heterocigosidad esperada: He, contenido de información polimórfica: PIC, equilibrio de Hardy-Weinberg: EHW. *Desvío significativo del equilibrio de Hardy-Weinberg $(\mathrm{P}<0.05)$

\begin{tabular}{lccccc}
\hline Locus & $\mathrm{Na}$ & $\mathrm{Ho}$ & $\mathrm{He}$ & $\mathrm{PIC}$ & $\mathrm{EHW}$ \\
\hline MiIIHR12 & 3.306 & 0.409 & 0.742 & 0.718 & $0.000^{* * *}$ \\
MiIIHR13 & 3.902 & 0.300 & 0.744 & 0.702 & $0.000^{* * *}$ \\
MiIIHR15 & 3.375 & 0.111 & 0.704 & 0.652 & $0.000^{* * *}$ \\
MiIIHR17 & 5.667 & 0.647 & 0.824 & 0.800 & $0.006^{* *}$ \\
MiIIHR18 & 3.945 & 0.214 & 0.768 & 0.729 & $0.000^{* * *}$ \\
MiIIHR19 & 4.167 & 0.500 & 0.760 & 0.730 & $0.020^{*}$ \\
MiIIHR23 & 7.918 & 0.412 & 0.874 & 0.862 & $0.000^{* * *}$ \\
MiIIHR24 & 6.081 & 0.533 & 0.836 & 0.815 & $0.000^{* * *}$ \\
MiIIHR26 & 5.885 & 0.059 & 0.832 & 0.810 & $0.000^{* * *}$ \\
MiIIHR31 & 2.569 & 0.053 & 0.611 & 0.573 & $0.000^{* * *}$ \\
MiIIHR34 & 2.117 & 0.211 & 0.528 & 0.493 & $0.000^{* * *}$ \\
MiIIHR36 & 5.684 & 0.778 & 0.824 & 0.802 & $0.001 * *$ \\
Promedio total & 4.551 & 0.355 & 0.748 & 0.724 & - \\
\hline
\end{tabular}

Para la heterocigosidad observada (Ho), los resultados obtenidos oscilaron entre 0.778 y 0.053 correspondientes a MiIIHR36 y MiIIHR31 respectivamente con un promedio de 0.355 ; mientras que los valores de heterocigosidad esperada (He) fueron de 0.874 y 0.528 , correspondientes a MiIIHR23 y MiIIHR34 respectivamente con un promedio de 0.748 (Cuadro 2).

El contenido de información polimórfica (PIC) promedio obtenido para la población fue de 0.724 y sus valores oscilaron entre 0.862 y 0.493 (Cuadro 2), los cuales corresponden a los marcadores MiIIHR23 y MiIIHR34 respectivamente.

Los 12 marcadores microsatélites estudiados mostraron ausencia del equilibrio de Hardy-Weinberg $(\mathrm{p}<0.05)$ (Cuadro 2).

El valor promedio del estadístico $\mathrm{F}_{\text {IS }}$ (Cuadro 3) fue de 0.345 , con valores que oscilan entre 0.014 para el locus MiIIHR24 y 0.907 para el locus MiIIHR26; con relación 
Cuadro 3: Valores de los estadísticos $F$ de Wright $\left(\mathrm{F}_{\mathrm{IS}}, \mathrm{F}_{\mathrm{IT}} \mathrm{F}_{\mathrm{ST}}\right)$ para cada marcador en la población de Valencia, Córdoba, Colombia.

\begin{tabular}{cccc}
\hline Locus & $\mathrm{F}_{\text {IS }}$ & $\mathrm{F}_{\text {IT }}$ & $\mathrm{F}_{\text {ST }}$ \\
\hline MiIIHR12 & 0.281 & 0.412 & 0.182 \\
MiIIHR13 & 0.525 & 0.682 & 0.331 \\
MiIIHR15 & 0.655 & 0.828 & 0.501 \\
MiIIHR17 & -0.322 & 0.185 & 0.383 \\
MiIIHR18 & 0.759 & 0.845 & 0.358 \\
MiIIHR19 & 0.183 & 0.456 & 0.335 \\
MiIIHR23 & 0.056 & 0.555 & 0.529 \\
MiIIHR24 & 0.014 & 0.547 & 0.540 \\
MiIIHR26 & 0.907 & 0.939 & 0.343 \\
MiIIHR31 & 0.784 & 0.905 & 0.559 \\
MiIIHR34 & 0.458 & 0.572 & 0.210 \\
MiIIHR36 & -0.158 & 0.051 & 0.180 \\
Media & 0.345 & 0.581 & 0.371 \\
\hline
\end{tabular}

al estadístico $\mathrm{F}_{\mathrm{IT}}$, el valor promedio fue de 0.581 , en rangos de 0.051 a 0.939 para los loci MiIIHR36 y MiIIHR26 respectivamente. Por otro lado, el valor promedio de $\mathrm{F}_{\mathrm{ST}}$ obtenido fue de 0.371 con valores que oscilan entre 0.180 para el locus MiIIHR36 y 0.559 para el locus MiIIHR31.

Atendiendo a los valores de distancia genética obtenidas (Cuadro 4), puede apreciarse que las subpoblaciones de Valencia y El Reposo resultaron ser las subpoblaciones más parecidas genéticamente y las más cercanas geográficamente, mientras que Villanueva y Santo Do- mingo fueron las subpoblaciones menos parecidas y las más alejadas geográficamente.

En el dendrograma generado para el análisis de las subpoblaciones (Fig. 2), se muestra la asociación que se presentó entre las subpoblaciones Villanueva, Valencia, El Reposo y San Rafael del Pirú, las cuales comparten su cercanía geográfica. Además, se muestra la subpoblación de Santo Domingo como la más alejada, siendo esta la más distante geográficamente.

\section{Discusión}

El número medio de alelos por locus obtenido en esta investigación fue menor al obtenido por Dinesh et al. (2015) con una media de 12 alelos por locus. La obtención de un número de alelos inferior en nuestro estudio respecto al reportado se puede explicar por un descenso en la variabilidad del material vegetal analizado debido a un menor número de genotipos diferentes, y fue mayor al reportado por dos Santos Ribeiro et al. (2012), ArmasMoreno (2013) y Azmat et al. (2016) quienes registraron un promedio de 4, 2.6 y 3 alelos respectivamente, lo cual muestra que el material vegetal del presente estudio posee un mayor número de genotipos diferentes. Por otro lado, fue similar al obtenido por Bajpai et al. (2016) quien reportó un valor promedio de seis alelos.

Según Botstein et al. (1980), 11 de los 12 loci utilizados en este trabajo presentan valores del PIC superiores al valor de 0.5 indicado como umbral por encima del cual un marcador puede ser considerado muy informativo al momento de detectar la variabilidad genética y utilizar-

Cuadro 4: Matriz de distancia genética de Nei (1972) en las subpoblaciones estudiadas en Valencia, Córdoba, Colombia.

\begin{tabular}{lccccc}
\hline & Villanueva & Valencia & El Reposo & San Rafael del Pirú & Santo Domingo \\
\hline Villanueva & ------- & & & & \\
Valencia & 0.830 & ------ & & & \\
El Reposo & 0.705 & 0.456 & ------- & & \\
San Rafael del Pirú & 0.983 & 1.031 & 0.982 & ----- & - \\
Santo Domingo & 1.951 & 1.592 & 1.343 & 1.508 & ---- \\
\hline
\end{tabular}




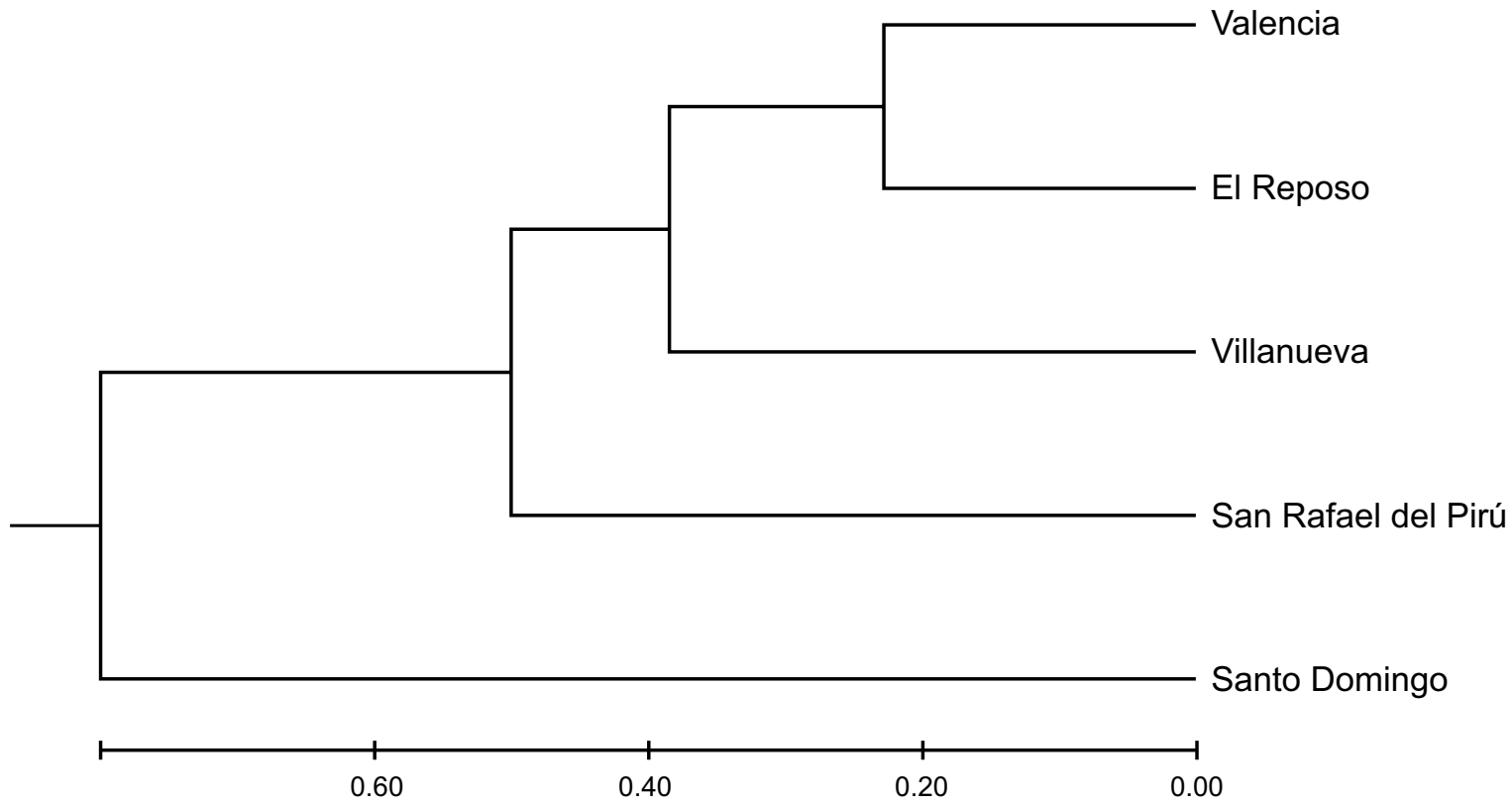

Figura 2: Dendograma basado en la distancia genética de Nei (1972) entre las subpoblaciones estudiadas en Valencia, Córdoba, Colombia, obtenido por el método UPGMA.

se eficazmente en estudios de diversidad genética en la población de mango estudiada. Nueve loci (MiIIHR12, MiIIHR13, MiIIHR17, MiIIHR18, MiIIHR19, MiIIHR23, MiIIHR24, MiIIHR26 y MiIIHR36) muestran un PIC superior a 0.7 , valor por encima del cual el marcador es especialmente de utilidad para la construcción de mapas genéticos (Bandelj et al., 2004), y solo uno medianamente informativo, con valor de PIC mayor a 0.25. El PIC promedio obtenido en el presente estudio, utilizando marcadores STRs, resultó mayor al reportado por Begum et al. (2012) ( $\mathrm{PIC}=0.66)$, Begum et al. (2013) ( $\mathrm{PIC}=0.46)$, Tasliah et al. (2013) ( $\mathrm{PIC}=0.548)$, Begum et al. (2014) ( $\mathrm{PIC}=0.47)$, Bajpai et al. (2016) $(\mathrm{PIC}=0.438)$, dos Santos Alves et al. (2016) ( $\mathrm{PIC}=0.550)$, Gitahi et al., (2016), (PIC=0.543) y Nazish et al. (2017) (PIC=0.398); menor al reportado por Ravishankar et al. (2015) $(\mathrm{PIC}=0.841) \mathrm{y}$ similar al reportado por Hirano et al. (2010) $(\mathrm{PIC}=0.716)$ y Surapaneny et al. (2013) (PIC=0.717).

Los resultados obtenidos para la heterocigosidad observada (Ho) fueron menores a los reportados por Viruel et al. (2005) $(\mathrm{Ho}=0.69)$, Gálvez-López et al. (2009) $(\mathrm{Ho}=0.51)$,
Hirano et al. (2010) $(\mathrm{Ho}=0.645)$, Ravishankar et al. (2015) $(\mathrm{Ho}=0.624)$, dos Santos Alves et al. (2016) $(\mathrm{Ho}=0.437)$ y Gitahi et al. (2016) $(\mathrm{Ho}=0.64)$, y los valores encontrados para la heterocigosidad esperada $(\mathrm{He})$ fueron mayores a los reportados por Viruel et al. (2005) $(\mathrm{He}=0.65)$, Hirano et al. (2010) $(\mathrm{He}=0.709)$, Surapaneny et al. (2013) $(\mathrm{He}=0.390)$, dos Santos Alves et al. (2016) $(\mathrm{He}=0.624)$, Gitahi et al. (2016) $(\mathrm{He}=0.55)$ y Nazish et al. (2017) $(\mathrm{He}=0.262)$, y menores a los reportados por Gálvez-López et al. (2009) $(\mathrm{He}=0.92)$, Ravishankar et al. (2015) $(\mathrm{He}=0.858)$.

El número de alelos efectivos $(\mathrm{Na})$ para la población de mango estudiada osciló entre 2.11 y 7.91 con un promedio de 4.55, valor representativo según Bakoumé et al. (2007), quienes proponen que se requiere de un alto número de alelos para la selección de individuos con caracteres deseables y evitar la pérdida de diversidad genética a través de planes de conservación. Este resultado $(\mathrm{Na}=4.55)$, es mayor al reportado por Viruel et al. (2005) $(\mathrm{Na}=3.3)$, Gitahi et al. (2016) $(\mathrm{Na}=3.0)$ y Nazish et al. (2017) $(\mathrm{Na}=1.8)$, y menor en comparación con el promedio obtenido por Hirano et al. (2011) $(\mathrm{Na}=6.5)$. 
Los 12 marcadores microsatélites mostraron una desviación significativa con respecto al equilibrio de Hardy-Weinberg, lo cual puede deberse a endogamia, existencia de una posible estructura genética por subdivisión (Efecto Wahlund), emparejamiento selectivo o poblaciones pequeñas (Chapuis y Estoup, 2007). También pudo haberse dado un efecto fundador, donde los pocos individuos que forman la nueva población no representan el perfil genético de la de origen, sumado a la fuerte recombinación que pudo darse entre los escasos individuos iniciales de la población (Nichols et al., 2001).

Los valores obtenidos para los estadísticos $\mathrm{F}_{\text {IS }} \mathrm{y}$ $\mathrm{F}_{\mathrm{IT}}$ fueron positivos para todos los marcadores revelando un déficit de heterocigotos. El exceso de homocigotos en una población podría ser el resultado de eventos de endogamia, lo cual puede aumentar la probabilidad de que se pierdan alelos para la siguiente generación, llevando a la pérdida progresiva de diversidad genética con el paso del tiempo (Ellegren y Galtier, 2016).

De otra parte, la cercanía genética resultante entre las subpoblaciones Villanueva, Valencia, E1 Reposo y San Rafael del Pirú concuerda con lo expuesto por Rocha y Lobo (1996), en su trabajo con Enterolobium cyclocarpum Jacq., en donde la distancia genética resultó directamente proporcional a la distancia geográfica.

\section{ConCLusiones}

El estudio de Mangifera indica en Valencia, Córdoba, Colombia, reveló una alta diversidad genética; además, presentó un elevado número de alelos y ausencia de equilibrio de Hardy-Weinberg. Los marcadores utilizados resultaron ser altamente informativos, atendiendo al PIC, razón por la cual son recomendables para posteriores análisis de diversidad genética en esta especie.

\section{CONTRIBUCIÓN DE AUTORES}

RR y EP concibieron, diseñaron el estudio, efectuaron los análisis e interpretaron los resultados. MG llevó a cabo los experimentos, realizó parte de los análisis y escribió el manuscrito con la ayuda de RR y EP. Todos los autores contribuyeron a la discusión, revisión y aprobación del manuscrito final.

\section{FINANCIAMIENTO}

Este estudio fue apoyado por la Universidad de Córdoba (Proyecto $N^{\circ}$ FCB-05-14).

\section{AgradeCIMIENTOS}

Los autores desean agradecer a Heidy Saab Ramos y Mauricio Begambre Hernández por el apoyo brindado para la realización de este trabajo.

\section{LITERATURA CITADA}

Abràmoff, D., J. Magalhães y J. Ram. 2004. Image processing with ImageJ. Biophotonics International 11(7): 36-42.

Adato, A., D. Sharon, U. Lavi, J. Hillel y S. Gazit. 1995. Application of DNA fingerprints for identification and genetic analyses of mango (Mangifera indica) genotypes. Journal of the American Society for Horticultural Science 120(2): 259-264.

Armas-Moreno, M. 2013. Caracterización molecular de 54 accesiones de guanábana (Annona muricata L.) y 60 de mango (Mangifera indica L.) a través de marcadores genéticos moleculares de las colecciones del banco de germoplasma del INIAP. Tesis de Ingeniería Agropecuaria. Facultad de Ciencias Agrarias, Universidad Estatal Península de Santa Elena. La Libertad, Ecuador. 110 pp.

Aron, Y., H. Czosnek, S. Gazit y C. Degani. 1997. Segregation distortion and linkage of mango isozyme loci. Horticulturae Scientia 32(5): 918-920.

Asohofrucol y Corpoica. 2013. Modelo tecnológico para el cultivo del mango en el valle del Alto Magdalena en el departamento del Tolima. http://www.asohofrucol.com. co/archivos/biblioteca/biblioteca_264__MP_Mango.pdf (consultado marzo de 2017).

Azmat, M., A. Khan, I. Khan, I. Ahmad, H. Cheema y A. Khan. 2016. Morphological characterization and SSR based DNA fingerprinting of elite commercial mango cultivars. Pakistan Journal of Agricultural Sciences 53(2): 321-330. DOI: https://dx.doi.org/10.21162/PAKJAS/16.2988

Azofeifa-Delgado, A. 2006. Uso de marcadores moleculares en plantas; aplicaciones en frutales del trópico. Mesoamerican Agronomy 17(2): 221-241. DOI: https:// doi.org/10.15517/am.v17i2.5163 
Bajpai, A., M. Muthukumar, I. Ahmad, K. V. Ravishankar, V. A. Parthasarthy, B. Sthapit, R. Rao, J.P. Vermay S. Rajan. 2016. Molecular and morphological diversity in locally grown non-commercial (heirloom) mango varieties of North India. Journal of Environmental Biology 37(2): 221-228.

Bakoumé, C., R. Wickneswari, N. Rajanaidu, A. K. Din, P. Amblard y N. Billotte. 2007. Diversidad alélica de poblaciones naturales de palma de aceite (Elaeis guineensis Jacq.) detectada por marcadores microsatelitales: Implicación en conservación. Palmas 28: 149-158.

Bandelj, D., J. Jakše y B. Javornik. 2004. Assessment of genetic variability of olive variety by microsatellite and AFLP markers. Euphytica 136(1): 93-102. DOI: https://doi. org/10.1023/b:euph. 0000019552.42066.10

Becerra, V. y M. Paredes. 2000. Uso de marcadores bioquímicos y moleculares en estudios de diversidad genética. Agricultural Technology 60(3): 270-281. DOI: https:// dx.doi.org/10.4067/S0365-28072000000300007

Begum, H., M. T. Reddy, S. Malathi, B. P. Reddy, S. Arcahk, J. Nagaraju y E. A. Siddiq. 2012. Molecular analysis for genetic distinctiveness and relationships of indigenous landraces with popular cultivars of mango (Mangifera indica L.) in Andhra Pradesh, India. The Asian and Australasian Journal of Plant Science and Biotechnology 6(1): 24-37.

Begum, H., M. T. Reddy, S. Malathi, B. P. Reddy, G. Narshimulu, J. Nagaraju y E. A. Siddiq. 2013. Microsatellite analysis of intracultivar diversity in 'Chinnarasam' mango from Andhra Pradesh India. African Crop Science Journal 21(2): 109-117.

Begum, H., M. T. Reddy, S. Malathi, P. Reddy, G. Narshimulu, J. Nagaraju y E. A. Siddiq. 2014. Morphological and microsatellite analysis of intravarietal variability in 'Cherukurasam' cultivar of mango (Mangifera indica L.). Jordan Journal of Agricultural Sciences 10(3): 452-471.

Botstein, D., R. L. White, M. Skolnick y R. W. Davis. 1980. Construction of a genetic linkage map in man using restriction fragment length polymorphisms. The American Journal of Human Genetics 32(3): 314-331.

Chapuis, M. P. y A. Estoup. 2007. Microsatellite null alleles and estimation of population differentiation. Molecular
Biology and Evolution 24(3): 621-631. DOI: https:// dx.doi.org/10.1093/molbev/ms1191

Degani, C., R. El-Batsri y S. Gazit. 1990. Enzyme polymorphisms in mango. Journal of the American Society for Horticultural Science 115(5): 844-847.

Díaz-Matallana, M., I. Schuler-García, M. Ruiz-García y E. Hodson-de-Jaramillo. 2009. Analysis of diversity among six populations of Colombian mango (Mangifera indica L. cvar. hilacha) using RAPDs markers. Electronic Journal of Biotechnology 12(3): 1-8. DOI: https://doi. org/10.2225/vol12-issue3-fulltext-10

Dinesh, M. R., K. V. Ravishankar, B. Sthapit, V. A. Parthasarathy, B. S. Sandya, P. Nischita y B. Lavanya. 2015. Genetic diversity studies in certain indigenous mango (Mangifera indica L.) varieties. Indian Journal of Plant Genetic Resources 28(1): 153-160. DOI: https:// dx.doi.org/10.5958/0976-1926.2015.00019.4

dos Santos Alves, E. O., F. P. Lima Neto, C. A. Fernandes Santos, I. C. N. dos Santos Ribeiro, C. A. Ferreira de Melo, I. S. Araújo Holanda, A. Pereira de Souza y R. X. Correa. 2016. Genetic diversity of mango accessions (Mangifera indica) using new microsatellite markers and morphological descriptors. Australian Journal of Crop Science 10(9): 1281-1287. DOI: https://dx.doi. org/10.21475/ajcs.2016.10.09.p7729

dos Santos Ribeiro, I. C. N., F. P. Lima Neto y C. A. F. Santos. 2012. Allelic database and accession divergence of a Brazilian mango collection based on microsatellite markers. Genetics and Molecular Research 12(4): 68026812. DOI: https://dx.doi.org/10.4238/2012.October.9.4

Eiadthong, W., K. Yonemori, S. Kanzaki y A. Sugiura. 2000. Amplified fragment length polymorphism analysis for studying genetic relationship among Mangifera species in Thailand. Journal of the American Society for Horticultural Science 125: 160-164.

Ellegren, H. y N. Galtier. 2016. Determinants of genetic diversity. Nature Reviews Genetics 17: 422-433. DOI: https://dx.doi.org/10.1038/nrg.2016.58

Espinal-García, C. F., H. J. Martínez-Covaleda y Y. PeñaMarín. 2005. La cadena de los frutales de exportación en Colombia: una mirada global de su estructura y dinámica 
1991-2005. Ministerio de Agricultura y Desarrollo Rural, Observatorio Agrocadenas Colombia. Documento de trabajo No. 67. Bogotá, Colombia. 68 pp.

Galal, O. A., H. A. Galal y A. A. Aboulila. 2017. Genetic variability and molecular characterization of some local and imported mango cultivars in Egypt. Egyptian Journal of Genetics and Cytology 46(1): 121-138.

Galán, V. 2009. El Cultivo del mango. Instituto Canario de Investigaciones agrarias. Gobierno de Canarias. 2da ed. Tenerife, España. 256 pp.

Gálvez-López D., S. Hernández-Delgado, M. González-Paz, E. N. Becerra-Leor, M. Salvador-Figueroa y N. MayekPérez. 2009. Genetic analysis of mango landraces from Mexico based on molecular markers. Plant Genetic Resources 7(3): 244-251.DOI: https://dx.doi.org/10.1017/ S147926210932434X

García, L. J., A. P. Sandoval, F. Forero, J. A. Floriano, G. Salamanca, J. A. Bernal, L. A. Vásquez y G. Gómez. 2010. Atributos de calidad del mango criollo para la agroindustria. Corporación Colombiana de Investigación Agropecuaria. Espinal, Colombia. 48 pp.

Gitahi, R., R. Kasili, M. Kyallo y K. Kehlenbeck. 2016. Diversity of threatened local mango landraces on smallholder farms in Eastern Kenya. Forests, Trees and Livelihoods 25(4): 239-254. DOI: https://dx.doi.org/10.1080/14728028.201 6.1201436

González, E. 2003. Microsatélites: sus aplicaciones en la conservación de la biodiversidad. Graellsia 59(2-3): 377388. DOI: https://doi.org/10.3989/graellsia.2003.v59.i23.253

Goudet, J., N. Perrin y P. Waser. 2002. Tests for sex-biased dispersal using bi-parentally inherited genetic markers. Molecular Ecology 11(6): 1103-1114. DOI: https://doi. org/10.1046/j.1365-294x.2002.01496.x

Hammer, O., D. A. T. Harper y P. D. Ryan. 2001. PAST: Paleontological statistics software package for education and data analysis. Palaeontologia Electronica 4(1): 1-9.

Hirano, R., T. Htun Oo y K. N. Watanabe. 2010. Myanmar mango landraces reveal genetic uniqueness over common cultivars from Florida, India, and Southeast Asia. Genome 53(4): 321-330. DOI: https://dx.doi.org/10.1139/g10-005
Hirano, R., H. Ishii, T. Htun Oo, S. A. Gilani, A. Kikuchi y K. N. Watanabe. 2011. Propagation management methods have altered the genetic variability of two traditional mango varieties in Myanmar, as revealed by SSR. Plant Genetic Resources 9(3): 404-410. DOI: https://doi.org/10.1017/ S1479262111000049

Hossain, M. A., M. M. Islam, M. A. Mannan, S. K. Roy y P. Shil. 2016. Molecular characterization of 25 Mango germplasm using RAPD markers available in the Southwestern Region of Bangladesh. International Journal of Bio-Resource and Stress Management 7(4): 807-813.

Human, C. y S. Rheeder. 2004. Mango breeding: results and successes. Acta Horticulturae 645: 331-335. DOI: https:// doi.org/10.17660/actahortic.2004.645.39

IPGRI. 2006. Descriptors for mango (Mangifera indica L.). International Plant Genetic Resources Institute. Rome, Italy. $60 \mathrm{pp}$.

Kalinowski, S. T., M. L. Taper y T. C. Marshall. 2007. Revising how the computer program CERVUS accommodates genotyping error increases success in paternity assignment. Molecular Ecology 16(5): 1099-1106. DOI: https://doi.org/10.1111/j.1365-294x.2007.03089.x

Kashkush, K., F. Jinggui, E. Tomer, J. Hillel y U. Lavi. 2001. Cultivar identification and genetic map of mango (Mangifera indica). Euphytica 122: 129-136.

Kessel, A. 2008. Aplicación de técnicas biotecnológicas en frutales, una vía valiosa para el rescate y la conservación de estas especies. Cultivos Tropicales 29: 27-37.

Krishna, H. y S. K. Singh. 2007. Biotechnological advances in mango (Mangifera indica $\mathrm{L}$.) and their future implication in crop improvement: a review. Biotechnology Advances 25(3): 223-243. DOI: https://doi.org/10.1016/j. biotechadv.2007.01.001

Kumar, S., G. Stecher y K. Tamura. 2016. MEGA7: Molecular Evolutionary Genetics Analysis version 7.0 for bigger datasets. Molecular Biology and Evolution 33(7): 18701874. DOI: https://doi.org/10.1093/molbev/msw054

Márquez, L., B. G. Pérez-Nievas, I. Gárate, B. García-Bueno, J. L. M. Madrigal, L. Menchén, G. Garrido y J. C. Leza. 2010. Anti-inflammatory effects of Mangifera indica $\mathrm{L}$. extract in a model of colitis. World Journal of 
Gastroenterology 16(39): 4922-4931. DOI: https://dx.doi. org/10.3748/wjg.v16.i39.4922

Nazish, T., G. Shabbir, A. Ali, S. Sami-ul-Allah, M. Naeem, M. Javed, S. Batool, A. Arshad, S. B. Hussain, K. Aslam, R. Seher, M. Tahir y M. Baber. 2017. Molecular diversity of Pakistani mango (Mangifera indica L.) varieties based on microsatellite markers. Genetics and Molecular Research 16(2): gmr16029560. DOI: https://dx.doi.org/10.4238/ gmr16029560

Nei, M. 1972. Genetic distance between populations. The American Naturalist 106(949): 283-92. DOI: https://doi. org/10.1086/282771

Nichols, R. A., M. W. Bruford y J. J. Groombridge. 2001. Sustaining genetic variation in a small population: evidence from the Mauritius kestrel. Molecular Ecology 10(3): 593-602. DOI: https://doi.org/10.1046/j.1365294x.2001.01204.x

Park, S. 2001. The Excel Microsatellite Toolkit. Animal Genomics Laboratory, University College. Dublin, Ireland.

Peakall, R. y P. Smouse. 2006. GenAlEx 6.5: genetic analysis in Excel: Population genetic software for teaching and research-an update. Bioinformatics 28(9): 2537-2539. DOI: https://doi.org/10.1093/bioinformatics/bts460

Pruthvish, R. y B. K. Chikkaswamy. 2016. Genetic diversity and relationships among Mango varieties using RAPD molecular markers. International Journal of Current Microbiology and Applied Sciences 5(1): 778-787. DOI: https://dx.doi.org/10.20546/ijcmas.2016.501.079

Qiu, S., J. Chen, S. Lin y X. Lin. 2012. A comparison of silver staining protocols for detecting DNA in polyester backed polyacrylamide gel. Brazilian Journal of Microbiology 43(2): 649-652. DOI: https://dx.doi.org/10.1590/S151783822012000200029

Ravishankar, K. V., B. H. Mani, L. Anand y M. R. Dinesh. 2011. Development of new microsatellite markers from Mango (Mangifera indica) and cross-species amplification. American Journal of Botany 98(4): e96-e99. DOI: https:// dx.doi.org/10.3732/ajb.1000263

Ravishankar, K. V., P. Bommisetty, A. Bajpai, N. Srivastava, B. H. Mani, C. Vasugi, S. Rajan y M. R. Dinesh. 2015.
Genetic diversity and population structure analysis of mango (Mangifera indica) cultivars assessed by microsatellite markers. Trees 29(3): 775-783. DOI: https://dx.doi.org/10.1007/s00468-015-1155-x

Rocha, O. J. y J. A. Lobo. 1996. Genetic variation and differentiation among five populations of the Guanacaste tree (Enterolobium cyclocarpum Jacq.) in Costa Rica. International Journal of Plant Sciences 157(2): 234-239. DOI: https://doi.org/10.1086/297342

Samal, K. C., R. C. Jena, S. S. Swain, B. K. Das y P. K. Chand. 2012. Evaluation of genetic diversity among commercial cultivars, hybrids and local mango (Mangifera indica L.) genotypes of India using cumulative RAPD and ISSR markers. Euphytica 185(2): 195-213. DOI: https://dx.doi. org/10.1007/s10681-011-0522-y

Santos, C. A. F., F. P. Lima Neto, M. A. Rodrigues y J. Costa. 2008. Similaridade genética de acessos de mangueira de diferentes origens geográficas avaliadas por marcadores AFLP. Revista Brasileira de Fruticultura 30(3): 736-740. DOI: https://dx.doi.org/10.1590/S010029452008000300029

Sennhenn, A., K. Prinz, J. Gebauer, A. Whitbread, R. Jamnadass y K. Kehlenbeck. 2014. Identification of mango (Mangifera indica L.) landraces from Eastern and Central Kenya using a morphological and molecular approach. Genetic Resources and Crop Evolution 61(1): 7-22. DOI: https://doi.org/10.1007/s10722-013-0012-2

Souza, I. G. B., S. E. S. Valente, F. B. Britto, V. A. B. Souza y P. S. C. Lima. 2011. RAPD analysis of the genetic diversity of mango (Mangifera indica) germplasm in Brazil. Genetic and Molecular Research 10(4): 3080-3089. DOI: https://doi.org/10.4238/2011.december.14.1

Surapaneni, M., L. R. Vemireddy, H. Begum, B. P. Reddy, C. Neetasri, J. Nagaraju y E. A. Siddiq. 2013. Population structure and genetic analysis of different utility types of mango (Mangifera indica L.) germplasm of Andhra Pradesh state of India using microsatellite markers. Plant Systematics and Evolution 299(7): 1215-1229. DOI: https://dx.doi.org/10.1007/s00606-013-0790-1

Tasliah, T., H. Rijzaani, T. Z. Hariyadi, S. Yuriyah, R. Rebin, M. Ma’sumah y T. S. Silitonga. 2013. Analisis keragaman 
genetik 161 aksesi mangga Indonesia Menggunakan marka mikrosatelit. Jurnal AgroBiogen 9(3): 125-134. DOI: https://doi.org/10.21082/jbio.v9n3.2013.p125-134

Tsang, V., K. Hancock, M. Wilson, D. Palmer, S. Whaley, J. Dougal y S. Kennedy. 1986. Enzyme-linked immune electro transfer blot technique (Western blot) for human T-lymphotropic virus type III/lymphadenopathyassociated virus (HTLV-III/LAV) antibodies. Centers for Disease Control. Procedural Guide. Immunology Series No. 15. Atlanta, USA.

Valadez, E. y G. Kahl. 2000. Huellas de ADN en genomas de plantas. Editorial Mundi Prensa. Chapingo, México. 147 pp.

Vieccelli, J., D. Siqueira, W. Bispo y L. Lemos. 2016. Characterization of leaves and fruits of mango (Mangifera indica L.) cv. Imbu. Revista Brasileira de Fruticultura 38(3): e-193. DOI: https://dx.doi.org/10.1590/010029452016193
Viruel, M. A., P. Escribano, M. Barbieri, M. Ferri y J. I. Hormaza. 2005. Fingerprinting, embryo type and geographic differentiation in mango (Mangifera indica L., Anacardiaceae) with microsatellites. Molecular Breeding 15(4): 383-393. DOI: https://dx.doi.org/10.1007/s11032004-7982-x

Wang, M., D. Ying, Q. Wang, L. Li y R. Zhang. 2016. Genetic Diversity Analysis and Fingerprint Construction of Major Mango Cultivars in China. Journal of Agricultural Science and Technology 17(6): 1289-1294.

Yamanaka, N., M. Hasran, D. H. Xu, H. Tsunematsu, S. Idris y T. Ban. 2006. Genetic relationship and diversity of four Mangifera species revealed through AFLP analysis. Genetic Resources and Crop Evolution 53(5): 949-954. DOI: https://doi.org/10.1007/s10722-004-6695-7 